\title{
Direct measurement of the Wigner function by photon counting
}

\author{
K. Banaszek, C. Radzewicz, and K. Wódkiewicz \\ Wydziat Fizyki, Uniwersytet Warszawski, Hoża 69, PL-00-681 Warszawa, Poland \\ J. S. Krasiński \\ Center for Laser and Photonics Research, Oklahoma State University, 413 NCR, Stillwater, OK 74078, USA
}

(February 1, 2008)

We report a direct measurement of the Wigner function characterizing the quantum state of a light mode. The experimental scheme is based on the representation of the Wigner function as an expectation value of a displaced photon number parity operator. This allowed us to scan the phase space point-by-point, and obtain the complete Wigner function without using any numerical reconstruction algorithms.

PACS Number(s): 42.50.Ar, 03.65.Bz

Among many representations of the quantum state, the Wigner function offers an appealing possibility to describe quantum phenomena using the classical-like concept of phase space [1]. The Wigner function provides complete information on the state of a system, and it allows one to evaluate any quantum observable by phase space integration with an appropriate Wigner-Weyl ordered expression.

Recently, the Wigner function has gained experimental significance due to the development of the optical homodyne tomography, a beautiful technique for measuring the quantum state of light pioneered by Smithey et al. [2] and further applied by Breitenbach et al. [3] In this method, rooted in the domain of image processing, the Wigner function is a natural representation of the quantum state reconstructed from experimental data. However, the route from raw experimental results to the Wigner function is not straightforward. First, a sample of homodyne events is collected and stored. Statistics of these events for a fixed local oscillator phase is described by a marginal projection of the Wigner function. In order to retrieve the complete Wigner function, a family of homodyne statistics measured for a sufficiently dense set of local oscillator phases has to be processed using the sophisticated filtered back-projection algorithm.

In this Communication we report a direct measurement of the Wigner function of a light mode. This technique, based on photon counting, avoids the detour via complex numerical reconstruction algorithms. The principle of our measurement is entirely different from optical homodyne tomography. The Wigner function at a given phase space point is itself a well defined quantum observable [4]. Furthermore, the measurement of this observable can be implemented for optical fields using an arrangement employing an auxiliary coherent probe beam [5,6]. The amplitude and the phase of the probe field define the point in the phase space at which the Wigner function is measured. This allowed us to scan the phase space point-by-point, simply by changing the parameters of the probe field. A variation of this idea has been applied by Leibfried et al. [7] to determine the vibrational state of a trapped ion. Here we present an experiment, which to the best of our knowledge is the first direct measurement of the Wigner function for optical fields.

Our experiment is based on the representation of the Wigner function at a complex phase space point denoted by $\alpha$ as the expectation value of the following operator:

$$
\hat{W}(\alpha)=\frac{2}{\pi} \sum_{n=0}^{\infty}(-1)^{n} \hat{D}(\alpha)|n\rangle\langle n| \hat{D}^{\dagger}(\alpha),
$$

where $\hat{D}(\alpha)$ is the displacement operator and $|n\rangle$ denote Fock states, $\hat{n}|n\rangle=n|n\rangle$. Thus, $\hat{W}(\alpha)$ has two eigenvalues: $2 / \pi$ and $-2 / \pi$, corresponding to degenerate subspaces spanned respectively by even and odd displaced Fock states. Practical means to translate this formula into an optical arrangement are quite simple [5].6]. The displacement transformation can be realized by superposing the measured field at a low-reflection beam splitter with a strong coherent probe beam. The value of the displacement $\alpha$ is equal in this setup to the reflected amplitude of the probe field. Furthermore, the projections on Fock states can be obtained by photon counting assuming unit quantum efficiency. These two procedures, combined together, provide a practical way to measure the Wigner function at an arbitrarily selected phase space point $\alpha$.

The experimental setup we used to measure the Wigner function is shown schematically in Fig. 1. In principle, it is a Mach-Zender interferometric scheme with the beams in two arms of the interferometer serving as the signal and the probe fields. An attenuated, linearly polarized (in the plane of Fig. 1) $632.8 \mathrm{~nm}$ beam from a frequency-stabilized single-mode He:Ne laser is divided by a low-reflection beam splitter BS1. The weak reflected beam is used to generate the signal field whose Wigner function will be measured. The state preparation stage consists of a neutral density filter ND and a mirror mounted on a piezoelectric translator PZT. With this arrangement, we are able to create pure coherent states with variable phase as well as their incoherent mixtures. Though these states do not exhibit nonclassical properties, they constitute a nontrivial family to demonstrate 
the principle of the method, which provides a complete characterization of both quantum and classical field fluctuations.

The strong beam leaving the beam splitter BS1 plays the role of the probe field with which we perform the displacement transformation $\hat{D}(\alpha)$. In order to scan the phase space one should be able to set freely its amplitude and phase, which define respectively the radial and the angular coordinates in the phase space. The amplitude modulation is achieved with a half-wave plate, a longitudinal Pockels cell EOM1, and a polarizer oriented parallel to the initial direction of polarization. The phase modulation is done with the help of an ADP crystal electrooptic phase modulator EOM2 on the signal field. This is completely equivalent to modulating the probe field phase, but more convenient for technical reasons: in this arrangement optical paths in both the arms of the Mach-Zender interferometer are approximately the same, and better overlap of the signal and the probe modes is achieved at the output of the interferometer.

The signal and the probe fields are interfered at a nearly completely transmitting beam splitter BS2 with the power transmission $T=98.6 \%$. In this regime, the transmitted signal field effectively undergoes the required displacement transformation. Spurious reflections that accompany the beam leaving the interferometer are removed using the aperture A. Finally, the transmitted signal is focused on an EG\&G photon counting module SPCM-AQ-CD2749, whose photosensitive element is a silicon avalanche diode operated in the Geiger regime. The overall quantum efficiency of the module specified by the manufacturer is $\eta \geq 70 \%$. The count rate is kept low in the experiment, and thus the chance of two or more photons triggering a single avalanche signal is very small, and the probability of another photon arriving during the detector dead time can be neglected. Under these assumptions, each pulse generated by the module corresponds to the detection of a single photon [8]. The pulses are acquired by a computer, which also controls the voltages applied to the electrooptic modulators. The interference visibility in our setup has been measured to be $v \geq 98.5 \%$, and the phase difference between the two arms was stable up to few percent over times of the order of ten minutes.

In Fig. 2 1 we depict the measured Wigner functions of the vacuum, a weak coherent state, and a phase diffused coherent state. The phase fluctuations were obtained by applying a $400 \mathrm{~Hz}$ sine waveform to the piezoelectric translator. For all the plots, the phase space was scanned on a grid defined by 20 amplitudes and 40 phases. The scaling of the radial coordinate is obtained from the average number of photons $n_{\mathrm{vac}}$ detected for the blocked signal path. Thus the graphs are parameterized with the complex variable $\beta=e^{i \varphi} n_{\text {vac }}^{1 / 2}$, where $\varphi$ is the phase shift generated by the phase modulator EOM2. At each selected point of the phase space, the photocount statistics $p_{n}(\beta)$ was determined from a sequence of $N=8000$ counting intervals, each $\tau=30 \mu$ s long. The duration of the counting interval $\tau$ defines the temporal envelope of the measured mode. The count statistics was used to evaluate the alternating sum

$$
\Pi(\beta)=\sum_{n=0}^{\infty}(-1)^{n} p_{n}(\beta),
$$

which, up to the normalization factor $2 / \pi$ is equal to the Wigner function of the measured state. Statistical variance of this result can be estimated by $\operatorname{Var}[\Pi(\beta)]=$ $\left\{1-[\Pi(\beta)]^{2}\right\} / N$ [9]. Thus, the statistical error of our measurement reaches its maximum value, equal to $1 / N^{1 / 2} \approx 1.1 \%$, when the value of the Wigner function is close to zero.

The Wigner functions of the vacuum and of the coherent state are Gaussians centered at the average complex amplitude of the field, and their widths characterize quantum fluctuations. It can be noticed that the measured Wigner function of the coherent state is slightly lower than that of the vacuum state. In the following, when discussing experimental imperfections, we shall explain this as a result of non-unit interference visibility. In the plot of the Wigner function of the phase diffused coherent state, one can clearly distinguish two outer peaks corresponding to the turning points of the harmonically modulated phase.

There are several experimental factors whose impact on the result of the measurement needs to be analyzed. First, there are losses of the signal field resulting from two main sources: the reflection from the beam splitter BS2 and, what is more important, imperfect photodetection characterized by the quantum efficiency $\eta$. Analysis of these losses shows [0, 6], that in such a case the alternating series evaluated from photocount statistics is proportional to a generalized, $s$-ordered quasidistribution function $W(\alpha ; s)$, with the ordering parameter equal $s=-(1-\eta T) / \eta T$.

In addition, the two modes interfered at the beam splitter BS2 are never matched perfectly. The effects of the mode mismatch can be discussed most thoroughly within the multimode theory [10]. Here, due to limited space, we shall present the main conclusions and briefly sketch the reasoning. Let us consider the normalized mode functions describing the transmitted signal field and the reflected probe field. The squared overlap $\xi$ of these two mode functions can be related to the interference visibility $v$ as $\xi=v /(2-v)$. In order to describe the effects of the mode mismatch, we will decompose the probe mode function into a part that precisely overlaps with the signal, and the orthogonal remainder. The amplitude of the probe field effectively interfering with the signal is thus multiplied by $\xi^{1 / 2}$, and the remaining part of the probe field contributes to independent Poissonian counts with the average number of detected photons equal $(1-\xi)|\beta|^{2}$. Consequently, the full count statistics is given by a convolution of the statistics generated by the interfering fields, and the Poissonian statistics of mismatched photons. A 
simple calculation shows, that the alternating sum evaluated from such a convolution can be represented as a product of the contributions corresponding to the two components of the probe field:

$$
\begin{aligned}
\Pi(\beta)= & \exp \left[-2(1-\xi)|\beta|^{2}\right] \\
& \times \frac{\pi}{2 \eta T} W\left(\sqrt{\frac{\xi}{\eta T}} \beta ;-\frac{1-\eta T}{\eta T}\right) .
\end{aligned}
$$

Here on the right-hand side we have made use of the theoretical results for imperfect detection obtained in Refs. [5]6]. Specializing this result to a coherent state $\left|\alpha_{0}\right\rangle$ with the amplitude $\alpha_{0}$, yields:

$$
\Pi(\beta)=\exp \left[-2\left|\beta-\sqrt{\xi \eta T} \alpha_{0}\right|^{2}-2(1-\xi) \eta T\left|\alpha_{0}\right|^{2}\right] .
$$

Thus, in a realistic case $\Pi(\beta)$ represents a Gaussian centered at the attenuated amplitude $\sqrt{\xi \eta T} \alpha_{0}$, and the width remains unchanged. This Gaussian function in multiplied by the constant factor $\exp \left[-2(1-\xi) \eta T\left|\alpha_{0}\right|^{2}\right]$. For our measurement, $\xi \approx 97 \%$ and $\eta T\left|\alpha_{0}\right|^{2} \approx 1.34$, which gives the value of this factor equal 0.92 . This result agrees with the height of the experimentally measured Wigner function of a coherent state.

We shall conclude this Communication with a brief comparison of the demonstrated direct method for measuring the Wigner function with the optical homodyne tomography approach. An important parameter in the experimental quantum state reconstruction is the detection efficiency. Currently, higher values of this parameter can be achieved in the homodyne technique, which detects quantum fluctuations as a difference between two rather intense fields. Such fields can be efficiently converted into photocurrent signals with the help of $p$ - $i$-n diodes. It should be also noted that an avalanche photodiode is not capable of resolving the number of simultaneously absorbed photons, and that it delivers a signal proportional to the light intensity only in the regime described in this paper. However, continuous progress in single photon detection technology gives hope to overcome current limitations of photon counting 11. Alternatively, the displacement transformation implemented in the photon counting technique can be combined with efficient random phase homodyne detection. This yields the recently proposed scheme for cascaded homodyning [12].

The simplicity of the relation (1) linking the count statistics with quasidistribution functions allows one to determine the Wigner function at a given point from a relatively small sample of experimental data. This feature becomes particularly advantageous, when we consider detection of multimode light. Optical homodyne tomography requires substantial numerical effort to reconstruct the multimode Wigner function. In contrast, the photon counting method has a very elegant generalization to the multimode case: after applying the displacement to each of the involved modes, the Wigner function at the selected point is simply given by the average parity of the total number of detected photons. Moreover, the dichotomic outcome of such a measurement provides a novel way of testing quantum nonlocality exhibited by correlated states of optical radiation [13].

The authors thank Prof. K. Ernst for placing a singlemode He:Ne laser at their disposal. This research is supported by Komitet Badań Naukowych, Grant 2P03B 00214.

[1] E. P. Wigner, Phys. Rev. 40, 749 (1932). For a review see M. Hillery, R. F. O'Connell, M. O. Scully, and E. P. Wigner, Phys. Rep. 106, 121 (1984).

[2] D. T. Smithey, M. Beck, M. G. Raymer, and A. Faridani, Phys. Rev. Lett. 70, 1244 (1993).

[3] G. Breitenbach, S. Schiller, and J. Mlynek, Nature 387, 471 (1997).

[4] A. Royer, Phys. Rev. A 15, 449 (1977); B.-G. Englert, J. Phys. A 22, 625 (1989); H. Moya-Cessa and P. L. Knight, Phys. Rev. A 48, 2479 (1993).

[5] S. Wallentowitz and W. Vogel, Phys. Rev. A 53, 4528 (1996).

[6] K. Banaszek and K. Wódkiewicz, Phys. Rev. Lett. 76, 4344 (1996).

[7] D. Leibfried, D. M. Meekhof, B. E. King, C. Monroe, W. M. Itano, and D. J. Wineland, Phys. Rev. Lett. 77, 4281 (1996).

[8] The operating mode of the SPCM results in a certain amount of extraneous pulses originating from dark counts and afterpulsing. The dark count rate of our module is less than $100 \mathrm{~s}^{-1}$. This gives on average $<3 \cdot 10^{-3}$ during a single counting interval, which is $30 \mu \mathrm{s}$ long. The typical afterpulsing probability is $0.2 \%$. Thus, both these effects give a negligible contribution to the measured count statistics.

[9] K. Banaszek and K. Wódkiewicz, J. Mod. Opt. 44, 2441 (1997).

[10] K. Banaszek et al., in preparation.

[11] P. G. Kwiat, A. M. Steinberg, R. Y. Chiao, P. H. Eberhard, and M. D. Petroff, Phys. Rev. A 48, R867 (1993).

[12] Z. Kis, T. Kiss, J. Janszky, P. Adam, S. Wallentowitz, and W. Vogel, Phys. Rev. A 59, R39 (1999).

[13] K. Banaszek and K. Wódkiewicz, Phys. Rev. Lett. 82, 2009 (1999). 


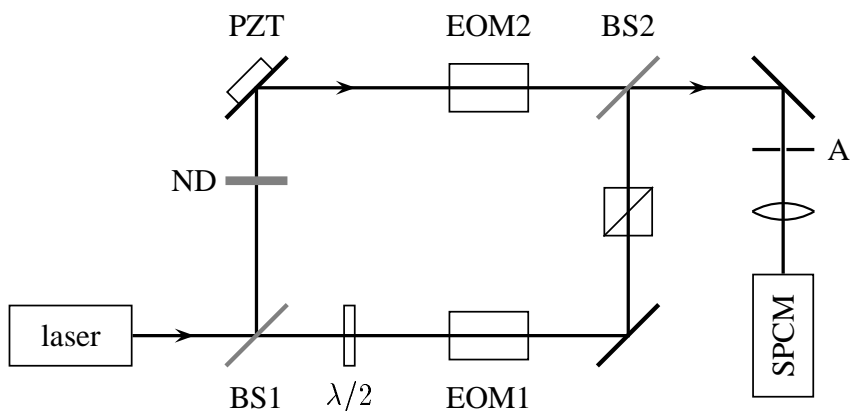

FIG. 1. The experimental setup for measuring the Wigner function. BS1 and BS2 are quartz plates serving as low-reflection beam splitters. The quantum state is prepared using the neutral density filter ND and a mirror mounted an a piezoelectric translator PZT. The electrooptic modulators EOM1 and EOM2 control respectively the amplitude and the phase of the point at which the Wigner function is measured. The signal field, after removing spurious reflections using the aperture A, is focused on a single photon counting module SPCM.

(a)
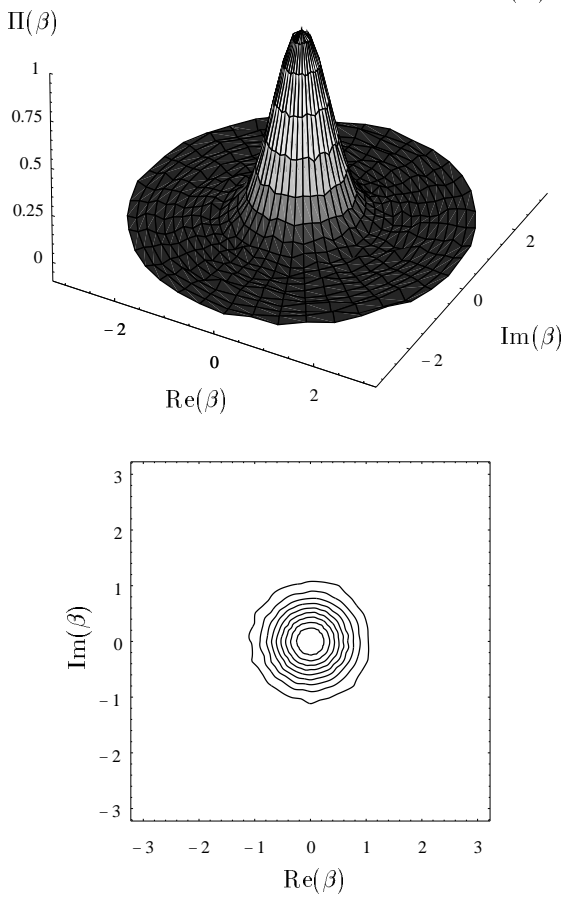

(b)
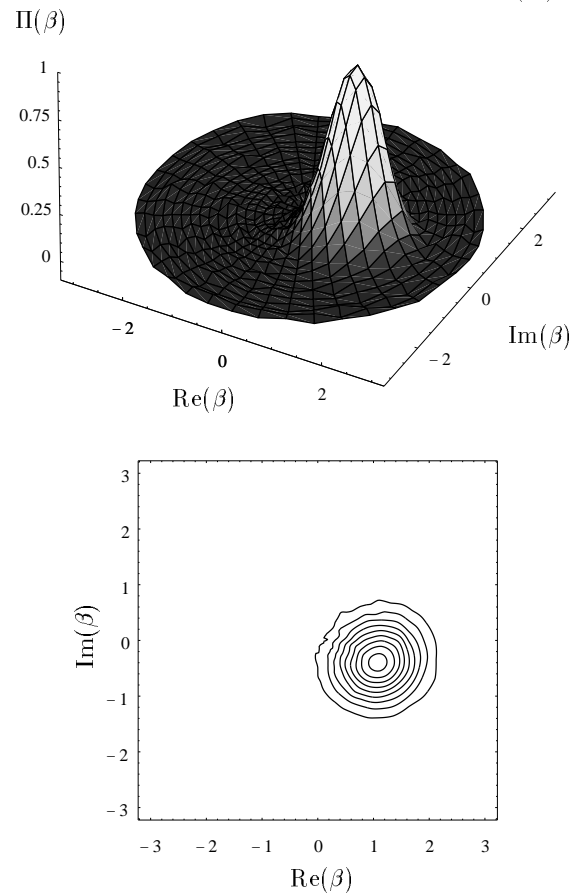

(c)
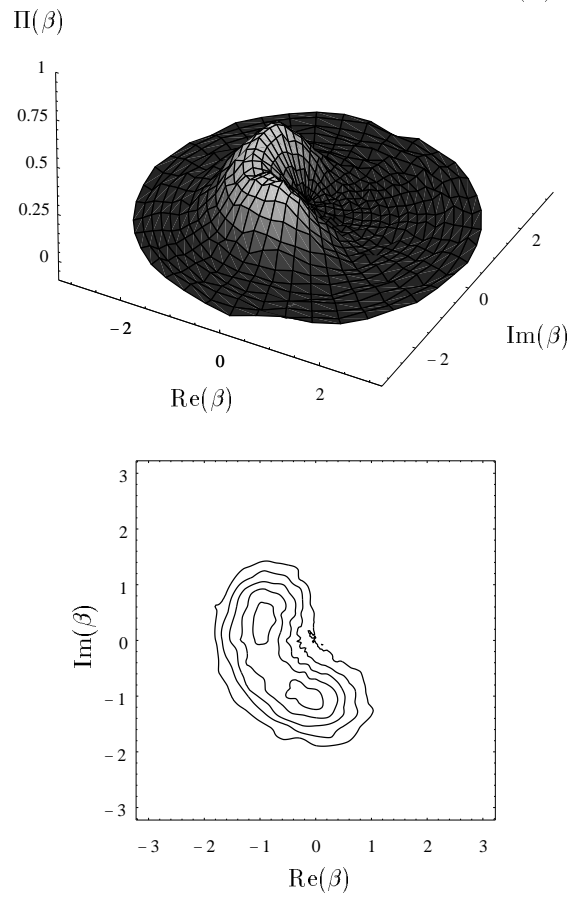

FIG. 2. The measured Wigner functions of (a) the vacuum, (b) a weak coherent state, and (c) a phase diffused coherent state. The contour plots depict interpolated heights given by multiples of 0.1 for the plots (a) and (b), and by $0.08,0.14,0.20,0.26,0.32$ for the plot $(\mathrm{c})$. 